\title{
Les herbicides halogénés de type phénoxyacides : évaluation clinico-biolo- gique des intoxications recensées au centre anti-poison d'Angers entre 1992 et 2005
}

\author{
Chlorophenoxyacid herbicides acute \\ poisoning : a 1992-2005 clinico-biological \\ retrospective study by the poison centre \\ in Angers
}

\section{Alain TURCANT ${ }^{(1) *}$, Catherine GANIERE-MONTEIL ${ }^{(2)}$, Anne LE BOUIL ${ }^{(1)}$, Laurence GAMELIN ${ }^{(3)}$, Patrick HARRY ${ }^{(3)}$}

(1) Service de Pharmacologie-Toxicologie, CHU Angers

(2) Service de Pharmacologie, CHU Hôtel-Dieu Nantes

(3) Centre Antipoison (CAP), CHU Angers

*Auteur à qui adresser la correspondance : Alain TURCANT, Service de Pharmacologie et Toxicologie, $\mathrm{CHU}, 4$, rue Larrey - 49933 ANGERS Cedex 9 - France Tél : 0241354552 - Fax : 0241354877 -E-mail : alturcant@chu-angers.fr

(Reçu le 16 février 2006; accepté le I9 juin 2006)

\section{RÉSUMÉ}

Six herbicides chlorophénoxyacides sont actuellement commercialisés en France le plus souvent en association entre eux: less acides 2,4-dichlorophénoxy-acétique (2,4-D), -propionique (2,4-DCP ou dichlorprop) ou -butyrique (2,4-DB), les acides 2-méthyl-4-chlorophényl-correspondants (MCPA, MCPP ou mécoprop, MCPB). L'acide 2,4,5-trichlorophénoxyacétique $(2,4,5-T)$ n'est plus autorisé actuellement. Ces produits, ainsi que le triclopyr; molécule voisine, peuvent se révéler très toxiques par ingestion. Les signes pronostiques

\section{SUMMARY}

Six chlorophenoxyacids herbicides are actually available in France :2,4-dichlorophenoxy acetic acid $(2,4-D)$ or its propionic (2,4-DCP or dichlorprop) or butyric $(2,4-D B)$ homologues and 2-methyl-4-chlorophenoxyacetic acid (MCPA) or the corresponding propionic (MCPP or mecoprop) or butyric (MCPB) acids. 2,4,5-trichlorophenoxyacetic $(2,4,5-T)$ is no longer available. Triclopyr is a structurally chemical analogue of these herbicides. Oral intake of these products can be very toxic with early symptoms as CNS depression, 
précoces de gravité sont des troubles de la conscience, une hypotension artérielle, une acidose métabolique. Une étude clinico-biologique rétrospective des intoxications par ces herbicides a été effectuée à partir des données du Centre AntiPoison d'Angers, entre 1992 et 2005, documentées par des dosages plasmatiques par clwomatographie en phase liquide. Chaque cas a été évalué selon les 5 classes (0 à 4) du score de gravité clinique PSS (Poison Severity Score). Vingt-sept cas d'intoxications volontaires $(n=27)$, dont 4 mortelles, ou accidentelles $(n=2)$ ont été recensés. Le 2,4 $D$ est retrouvé 14 fois, le 2,4,5-T 4 fois, le MCPA 11 fois, le MCPP 9 fois, le 2,4-DCP 8 fois et le triclopyr 4 fois soit près de deux molécules par patient. Les concentrations plasmatiques cumulées sont égales à $157 \pm 134 \mathrm{mg} / \mathrm{L}(11-350, n=$ 9) pour le groupe $P S S=0,514 \pm 306 \mathrm{mg} / \mathrm{L}(70-1110, n=$ 12) pour $P S S=1,867 \pm 273 \mathrm{mg} / \mathrm{L}(674-1060, n=2)$ pour $P S S=2$ et $844 \pm 525 \mathrm{mg} / L(529-1450, n=4)$ pour $P S S=4$. Les concentrations des cas mortels sont supérieures à 500 $\mathrm{mg} / \mathrm{L}$ mais des concentrations aussi élevées ont été observées chez des patients (PSS $\leq 2)$ guéris mais alcalinisés précocement. Quatre cinétiques réalisées montrent des demi-vies d'élimination variant entre 3-8 h $(2,4-D, 2,4,5-T)$ et 8-16 h (2,4-DCP, MCPA, MCPP) selon l'alcalinisation jugée par le $p H$ urinaire. Les concentrations plasmatiques doivent être interprétées avec prudence en tenant compte du pH. Tous les décès ont une acidose métabolique. Des concentrations supérieures à $100 \mathrm{mg} / \mathrm{L}$ entraînent des symptômes cliniques plus ou moins importants et des valeurs supérieures à 500 mg/L sont considérées comme potentiellement mortelles. Cependant aucune relation évidente entre concentration et effet n'est démontrée dans cette étude.

\section{MOTS-CLÉS}

Phénoxyacides, phytohormones, herbicides, intoxication, plasma.

\section{Introduction}

Les chlorophénoxyacides (fig. 1a) sont commercialisés depuis de nombreuses années comme herbicides à action systémique tant pour un usage professionnel (désherbage des semences) que domestique (allées et jardins). Ils agissent, après pénétration foliaire, comme l'Acide Indole Acétique (IAA) qui est une auxine naturelle, en stimulant mais de façon anarchique la croissance de la plante. De ce fait, ces herbicides sont également appelés auxines de synthèse ou encore phytohormones. A ce jour, six substances sont toujours commercialisées en France : quatre acides phénoxy-acétiques et deux acides phénoxy-butyriques avec différentes substitutions par un ou plusieurs atomes de chlore et un groupement méthyle, l'acide 2,4,5-trichlorophénoxyacétique n'étant plus autorisé (tableau I). Le triclopyr, où le cycle phényle est remplacé par un noyau pyridine (fig. Ib), est apparenté à cette famille. Ces produits sont souvent commercialisés en association par deux ou encore avec d'autres herbicides comme le fluroxypyr (analogue du triclopyr), le clopyralid (dérivé arterial hypotension or metabolic acidosis. A retrospective study of the acute poisoning registered by the Angers poison centre between 1992 and 2005 and documented by plasma levels is presented. The 27 cases (2 accidental and 25 selfpoisoning including 4 deaths) were graded using Poison Severity Score (0-4 scale). Detected molecules were 2,4-D $(n=14), 2,4,5-T(n=4), M C P A(n=11), M C P P(n=9)$, 2,4-DCP $(n=8)$ and triclopyr $(n \doteq 4)$ and represented near two products by poisoning. The sum of plasma levels were $157 \pm 134 \mathrm{mg} / \mathrm{L}$ (range $11-350, n=9$ ) for the $P S S=0$ group, $514 \pm 306 \mathrm{mg} / \mathrm{L}(70-1110, n=12)$ for $P S S=1,867 \pm 273$ $m g / L(674-1060, n=2)$ for $P S S=2$ and $844 \pm 525 m g / L$ $(529-1450, n=4)$ for $P S S=2$. Lethal levels were always more than $500 \mathrm{mg} / \mathrm{L}$ but higher levels were observed in PSS 2 cases who recovered with early alkalinization. Four kinetic data showed elimination half-lives between 3 and $8 h$ (2,4-D or 2,4,5-T) and 8-16h (2,4-DCP, MCPA, MCPP) according to alkalinization and urine $p H$. Metabolic acidosis was observed in all fatal cases. Initial plasma levels should be carefully elaluated with blood and urine pH. Minor or moderate symptoms are associated with plasma levels more than $100 \mathrm{mg} / \mathrm{L}$ while higher concentrations ( $\geq 500 \mathrm{mg} / \mathrm{L}$ ) are generally considered as potentially lethal. However, no evident relation between plasma level and clinical effect can be established in this study.

\section{KEY-WORDS}

Chlorophenoxyacids, herbicides, acute poisoning, plasma levels

de l'acide picolinique) ou le dicamba (dérivé de l'acide benzoïque). Ainsi, le catalogue des produits phytopharmaceutiques du ministère de l'agriculture et de la pêche fait état de 213 spécialités contenant de l'acide 2,4-dichlorophénoxyacétique (2,4-D) sous forme d'esters $(n=41)$ ou de sels généralement d'amines ( $\mathrm{n}=172$ ), de 169 spécialités contenant de l'acide 2-méthyl-4-chlorophénoxyacétique (MCPA ; esters $\mathrm{n}=23$, sels $\mathrm{n}=146$ ), de 167 spécialités contenant de l'acide 2-méthyl-4-chlorophénoxypropionique (MCPP ; esters $n=41$, sels $n=126$ ) ou encore de 38 spécialités à base de dichlorprop et 55 à base de triclopyr et seulement 2 spécialités d'acide 2-méthyl-4-chlorophénoxybutyrique (MCPB) et une d'acide 2,4-dichlorophénoxybutyrique $(2,4-\mathrm{DB})$.
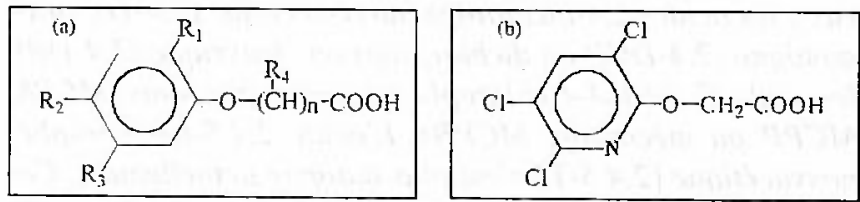

Figure 1 : Formules chimiques des phénoxyacides (a) et du triclopyr (b). 
Tableau I : Dénomination des différents herbicides phénoxyacides et apparentés (cf formules figure 1).

\begin{tabular}{|l|c|c|c|c|c|}
\hline Ac. Phénoxy- & $\mathrm{RI}$ & $\mathrm{R} 2$ & $\mathrm{R} 3$ & $\mathrm{R} 4$ & $\mathrm{n}$ \\
\hline acétiques & $\mathrm{Cl}$ & $\mathrm{Cl}$ & $\mathrm{H}$ & $\mathrm{H}$ & 1 \\
2,4-D (2,4-dichloro-) & $\mathrm{Cl}$ & $\mathrm{Cl}$ & $\mathrm{Cl}$ & $\mathrm{H}$ & 1 \\
2,4,5-T (2,4,5-trichloro-) & $\mathrm{H}$ & 1 \\
MCPA (2-méthyl-4-chloro-) & $\mathrm{CH}_{3}$ & $\mathrm{Cl}$ & $\mathrm{H}$ & $\mathrm{H}$ & 1 \\
\hline propioniques & & & & & \\
2,4-DCP (Dichlorprop) & $\mathrm{Cl}$ & $\mathrm{Cl}$ & $\mathrm{H}$ & $\mathrm{CH}_{3}$ & 1 \\
MCPP (Mécoprop) & $\mathrm{CH}_{3}$ & $\mathrm{Cl}$ & $\mathrm{H}$ & $\mathrm{CH}_{3}$ & 1 \\
\hline butyriques & & & $\mathrm{H}$ & $\mathrm{H}$ & 3 \\
2,4-DB & $\mathrm{Cl}$ & $\mathrm{Cl}$ & $\mathrm{H}$ & $\mathrm{H}$ & 3 \\
MCPB & $\mathrm{CH}_{3}$ & $\mathrm{Cl}$ & $\mathrm{H}$ & $\mathrm{H}$ & 1 \\
\hline Triclopyr (cycle pyridine) & $\mathrm{Cl}$ & $\mathrm{Cl}$ & $\mathrm{Cl}$ & $\mathrm{H}$ & \\
\hline
\end{tabular}

Si la toxicité par absorption cutanée est faible, il n'en est pas de même lors d'ingestion puisque les DL50 chez le rat sont comprises entre 400 et $1200 \mathrm{mg} / \mathrm{kg}$. Les doses potentiellement mortelles chez l'homme varient de 80 et $800 \mathrm{mg} / \mathrm{kg}$ ce qui correspond à une quantité de quelques grammes à quelques dizaines de grammes ingérés (1).

Le mécanisme de toxicité de ces herbicides reste mal connu. Cependant Bradberry et al. $(2,3)$ ont décrit l'implication possible de 3 mécanismes :

1) Une modification dose-dépendante de la structure des membranes (notamment au niveau de la barrière hématoencéphalique) ainsi qu'un blocage des systèmes de transport (anions organiques) au niveau de cette membrane pourraient expliquer la toxicité sur le système nerveux central ;

2) Une interférence par analogie structurale avec l'acide acétique ou avec l'acétylcholine conduirait respectivement à la formation de dérivés phénoxy-CoA $(2,4 \mathrm{D}-$ $\mathrm{CoA}, \ldots)$ et d'esters de choline (2,4-D-Choline, ...). Ceux-ci pourraient agir comme des faux messagers sur les récepteurs de l'acétylcholine;

3) Ces désordres métaboliques et la modification structurale des membranes conduiraient à une toxicité mitochondriale avec altération du transport d'électrons et un découplage de la phosphorylation oxydative suivi d'une déplétion en adénosine triphosphate (ATP).

Les données cinétiques montrent une absorption rapide et complète, une forte fixation à l'albumine plasmatique avec une saturation possible de cette liaison et une augmentation de la fraction libre lorsque la concentration d'herbicide est élevée. L'élimination est essentiellement urinaire sous forme libre. Une sécrétion active utilisant le système de transport des anions organiques explique, dans les cas d'exposition à de fortes doses, une saturation de l'élimination avec une cinétique de type Michaelis-Menten (4). De ce fait, la demi-vie pour ces substances, généralement comprise entre 20 et 40 heures, peut être beaucoup plus importante $(>200 \mathrm{~h}$ ) dans les cas d'intoxication massive (5).
Les principaux signes cliniques de gravité sont les troubles de la conscience, l'hypotension artérielle et l'acidose métabolique. Le traitement préconisé en cas d'intoxications repose sur une diurèse alcaline permettant d'augmenter la clairance d'un facteur 5 à 10 voire plus et entraîne ainsi une réduction des demi-vies à moins de 5 heures (5).

Nous présentons une étude rétrospective clinico-biologique des intoxications aiguës, recensées au Centre Antipoison d'Angers entre1992 et 2005 et documentées par des dosages sanguins.

\section{Matériel et Méthodes}

La gravité des intoxications aiguës par ces herbicides a été évaluée selon le Poison Severity Score ou PSS (6), qui comporte 5 stades allant de l'absence de signes cliniques $(=0)$ jusqu'au décès éventuel $(=4)$ en passant par l'augmentation progressive des différents symptômes. Cette gravité clinique a été comparée aux résultats des dosages d'herbicides. Ces dosages plasmatiques (et, dans quelques cas, urinaires) ont été faits à partir d'un faible volume d'échantillon $(100 \mu \mathrm{l})$ par simple précipitation des protéines par l'acétonitrile suivie d'une dilution au 1/2 dans l'eau avant analyse par chromatographie en phase liquide haute performance avec identification spectrale par détection UV à barrette de diodes (7). Pour 3 dossiers, une extraction liquide-liquide par l'éther en milieu acide a précédé la chromatographie liquide avec détection UV par barrette de diodes. Quelques cas d'intoxications ont été documentés par plusieurs dosages plasmatiques et urinaires afin de mieux évaluer la toxicocinétique de ces produits notamment après mise en place du traitement par alcalinisation.

\section{Résultats}

Vingt-sept cas (21 H - 6 F ; 3-88 ans) ont été recensés. A part 2 cas d'ingestion accidentelle par des enfants, iI s'agit d'intoxications volontaires dont 4 seront mortelles. Parmi les 6 produits détectés, le 2,4-D est la molécule la plus fréquente $(n=14)$ puis le MCPA $(n=11)$, le MCPP $(n=9)$, le dichlorprop $(n=8)$ et le $2,4,5$-T et le triclopyr $(n=4)$ soit près de 2 molécules par intoxication. Les concentrations sont très diverses et comprises entre 6 et $850 \mathrm{mg} / \mathrm{L}$ et les délais supposés entre ingestion et prise de sang sont relativement brefs $(<6$ h) sauf un ou deux cas tardifs.

En raison de la présence pratiquement constante de 2 phénoxyacides, la représentation des concentrations en herbicides en fonction du PSS (fig.2 et Tableau II) a été faite sur la somme des concentrations de chaque molécule. Aucune relation évidente n'apparaît même si les 
concentrations totales associées à une gravité 0 semblent plus faibles avec une moyenne de $157 \pm 134 \mathrm{mg} / \mathrm{L}$ et ne dépassent pas $350 \mathrm{mg} / \mathrm{L}$. La dispersion des valeurs pour le score de gravité 1 (70 à $1110 \mathrm{mg} / \mathrm{L}$ ) montre également la difficulté d'interprétation qui peut dépendre de l'herbicide impliqué (la DL50 orale rat du 2,4-D est la plus faible) mais aussi de la précocité de la prise en charge. Ainsi le cas présentant la valeur totale la plus élevée (1110 $\mathrm{mg} / \mathrm{L})$ correspond à la présence de MCPP ( $870 \mathrm{mg} / \mathrm{L})$, herbicide moins toxique que le 2,4-D $(240 \mathrm{mg} / \mathrm{L})$, et à une prise en charge rapide $2 \mathrm{~h}$ après l'ingestion. Néanmoins, pour les cas les plus graves, des valeurs supérieures à $500 \mathrm{mg} / \mathrm{L}$ sont généralement observées.

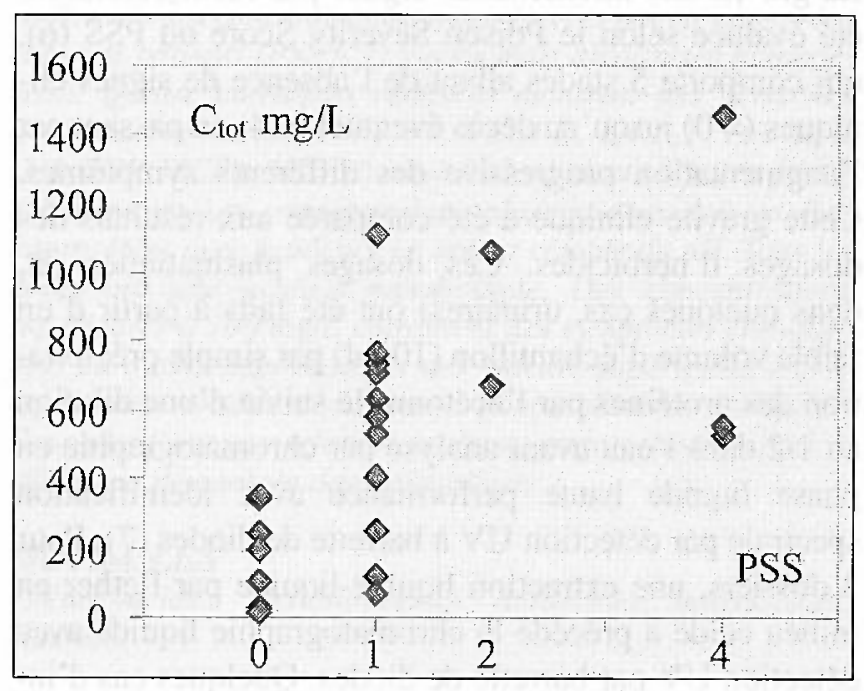

Figure 2 : Représentation des concentrations totales ( $m g / L$ ) des phénoxyacides observées dans les 27 cas d'intoxication en fonction du score de gravité clinique (PSS).

Tableau II : Concentrations des différents herbicides phénoxyacides et apparentés en fonction du score de gravité clinique (PSS).

\begin{tabular}{|c|c|c|c|}
\hline PSS & $\begin{array}{c}{\left[\mathbf{C}_{\text {tot }{ }^{\mathbf{m g} / \mathbf{L}}}\right.} \\
\mathbf{m}( \pm \mathbf{s})\end{array}$ & $\begin{array}{c}\mathrm{C}_{\mathbf{m i n}} \mathbf{C}_{\mathbf{m a x}} \\
\mathbf{m g} / \mathbf{L}\end{array}$ & n \\
\hline 0 & $157(134)$ & $11-350$ & 9 \\
\hline 1 & $514(306)$ & $70-1110$ & 12 \\
\hline 2 & $867(273)$ & $674-1060$ & 2 \\
\hline 4 & $844(525)$ & $529-1450$ & 4 \\
\hline
\end{tabular}

Les cas mortels (Tableau III) ont tous en commun des doses supposées ingérées importantes, une acidose métabolique modérée, des troubles de conscience très prononcés et un état de choc. Les décès, survenus en 12 à $72 \mathrm{~h}$, sont pour la plupart liés à un état de choc postanoxique. Le $1^{\text {tr }}$ cas, en fait le plus ancien (1993) et pour lequel seule une détection qualitative avait été réalisée dans le plasma, correspond à l'ingestion supposée de
$150 \mathrm{ml}$ d'un mélange de 2,4-D et de MCPP respectivement à 100 et $400 \mathrm{~g} / \mathrm{L}$. Le $2^{2}$ cas correspond à une intoxication massive par $500 \mathrm{ml}$ de LONPAR $($ qui est un mélange de 2,4-D et de MCPA mais aussi de clopyralid aux concentrations respectives de 150,175 et $35 \mathrm{~g} / \mathrm{L}$ (8). Le solvant est un mélange d'eau, de diméthylamine et d'éthanolamine. Les concentrations plasmatiques de 2,4$\mathrm{D}$ et de MCPA sont d'emblée très importantes (supérieures à $700 \mathrm{mg} / \mathrm{L} 4 \mathrm{~h}$ après l'ingestion). Le délai de prise en charge médicale, très court (1h) n'a pas empêché le décès du patient à la $26^{\circ}$ heure. Le $3^{\circ}$ cas est consécutif à l'absorption d'environ $220 \mathrm{ml}$ d'un désherbant total pour allées de parc, jardin et trottoir contenant un mélange de dichlorprop, de MCPA et de sulfosate respectivement à 54,54 et 93,6 g/L (9). Le sulfosate est un herbicide de la famille des amino-phosphonates qui sont des dérivés d'acides aminés. Ce produit ne diffère de son homologue, le glyphosate, que par la nature du contreion diméthylsulfonium au lieu de l'isopropylammonium. Ce produit n'a pas été quantifié mais a certainement contribué à la toxicité du mélange (DL50 chez le rat 748 $\mathrm{mg} / \mathrm{kg}$ ). Ni le délai de prise en charge très rapide (1h), ni l'instauration de la diurèse alcaline qui a permis de faire diminuer les concentrations plasmatiques, n'ont permis de sauver la patiente, l'anoxie cérébrale ayant précédé la prise en charge. Dans le $4^{c}$ cas, le produit absorbé est la DESORMONE®, mélange de 2,4-D et de dichlorprop, sous forme de sel de diméthylamine, à 130 et $520 \mathrm{~g} / \mathrm{L}$ mais le volume supposé ingéré est inconnu (10).

Sur ces 27 cas, quelques cinétiques ont pu être effectuées et les résultats sont les suivants : dans le cas $n^{\circ} 3$ d'intoxication mortelle par MCPA et dichlorprop (fig. 3), l'alcalinisation précoce s'est accompagnée d'une augmentation transitoire des concentrations, qui pourrait être expliquée par une réduction du volume de distribution, puis par une diminution de la demi-vie $(8$ à $10 \mathrm{~h}$ selon le produit). Dans la figure 4 est présenté un cas d'intoxication non mortelle où un traitement par alcalinisation importante et précoce a été pratiqué (dose de charge de $125 \mathrm{ml}$ de bicarbonate $4,2 \%$ soit $60 \mathrm{meq}$ puis 500 meq en $48 \mathrm{~h}, \mathrm{pH}$ urinaire toujours supérieur à 8,5 ). Les demi-vies de MCPA et MCPP, relativement parallèles, sont voisines de $24 \mathrm{~h}$ et diminuent progressivement à 7 ou $12 \mathrm{~h}$ selon le produit. Les demi-vies courtes (3 à 6h) observées dans un cas d'intoxication bénigne (fig. 5) sont sans doute en relation avec les concentrations initiales (h3) relativement faibles de $2,4-\mathrm{D}(155 \mathrm{mg} / \mathrm{L})$ et de 2,4,5-T (92 mg/L) et le $\mathrm{pH}$ des urines, d'emblée, très alcalin ( $>9)$. La clairance rénale, calculée dans ce dernier cas à partir des recueils d'urines toutes les 3 h, augmente progressivement de 14 à $81 \mathrm{ml} / \mathrm{min}$ pour le 2,4-D et de 6 à $49 \mathrm{ml} / \mathrm{min}$ pour le $2,4,5-\mathrm{T}$ entre la $3^{c}$ et la $15^{\circ}$ heure. La figure 6 illustre l'importance de cette alcalinisation puisque l'élimination apparaît plus lente pendant les 10 premières heures où le $\mathrm{pH}$ urinaire est inférieur à 6 (T1/2 
Tableau III : Caractéristiques des 4 cas mortels d'intoxications par herbicides phénoxyacides.

\begin{tabular}{|c|c|c|c|c|c|c|c|c|c|c|}
\hline $\begin{array}{c}\text { Cas } \\
n^{\circ}\end{array}$ & Sexe & $\begin{array}{l}\text { Age } \\
\text { (ans) }\end{array}$ & $\begin{array}{c}\text { Dose } \\
\text { supposée }\end{array}$ & pH & GS & $\begin{array}{c}+ \\
\text { (h) }\end{array}$ & $\begin{array}{c}, 4-\mathrm{D} \\
(\mathrm{mg} / \mathrm{L}) \\
\end{array}$ & $\begin{array}{l}\text { MCPA } \\
(\mathrm{mg} / \mathrm{L})\end{array}$ & $\begin{array}{l}\text { MCPP } \\
\text { (mg/L) }\end{array}$ & $\begin{array}{c}\mathbf{D C P} \\
(\mathrm{mg} / \mathrm{L})\end{array}$ \\
\hline 1 & $\mathrm{H}$ & 47 & $15-60 \mathrm{~g}$ & 7,28 & 6 & 12 & $\mathrm{P}+$ & & Pt & \\
\hline 2 & $\begin{array}{c}\mathrm{H} \\
\text { (ref. } 8 \text { ) }\end{array}$ & 88 & $75-87 \mathrm{~g}$ & 7,28 & 3 & 26 & $\begin{array}{l}700^{\mathrm{a}} \\
370^{\mathrm{b}}\end{array}$ & $\begin{array}{l}750 \\
340\end{array}$ & & \\
\hline 3 & $\begin{array}{c}F \\
\text { (ref. 9) }\end{array}$ & 47 & $12-12 \mathrm{~g}$ & 7,29 & 3 & 72 & & $\begin{array}{l}246^{c} \\
249^{d}\end{array}$ & & $\begin{array}{l}283 \\
293\end{array}$ \\
\hline 4 & $\begin{array}{c}\overline{\mathrm{H}} \\
\text { (ref. 10) }\end{array}$ & 51 & $\begin{array}{c}130-520 \\
\mathrm{~g} / \mathrm{L}\end{array}$ & 7,26 & $8-3$ & 46 & $377^{\mathrm{e}}$ & & & 175 \\
\hline
\end{tabular}

GS : Glasgow coma Score ; $†$ : délai du décès par rapport à l'ingestion ; P+: présence dans le plasma ; a-e : délai supposé entre ingestion et prélèvement ( $a: h 4 ; b: h 26 ; c: h 5 ; d: h 17 ; e: h 24)$.

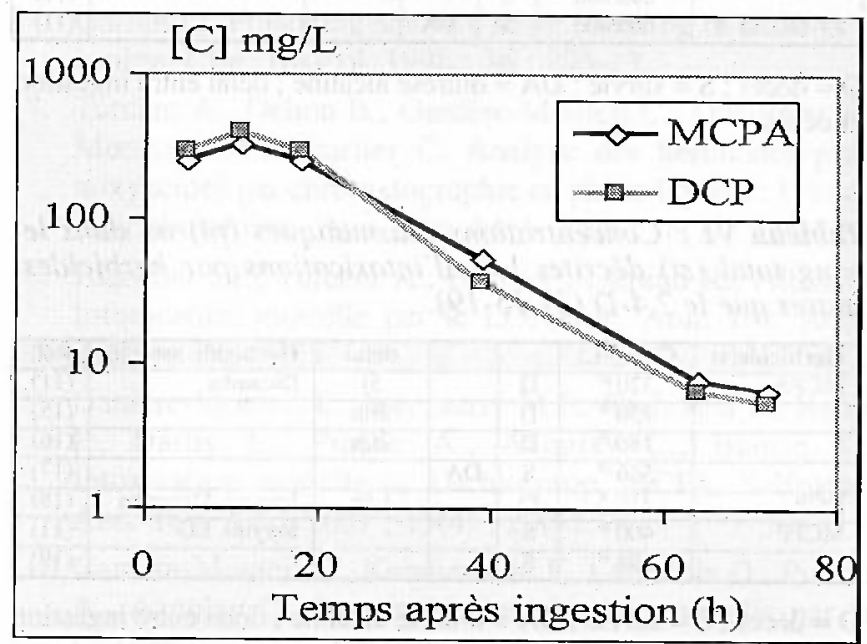

Figure 3 : Cinétiques du MCPA et DCP observées dans un cas d'intoxication mortelle ( $\left.n^{\circ} 3\right)$ après ingestion de $12 \mathrm{~g}$ de chaque produit et alcalinisation précoce.

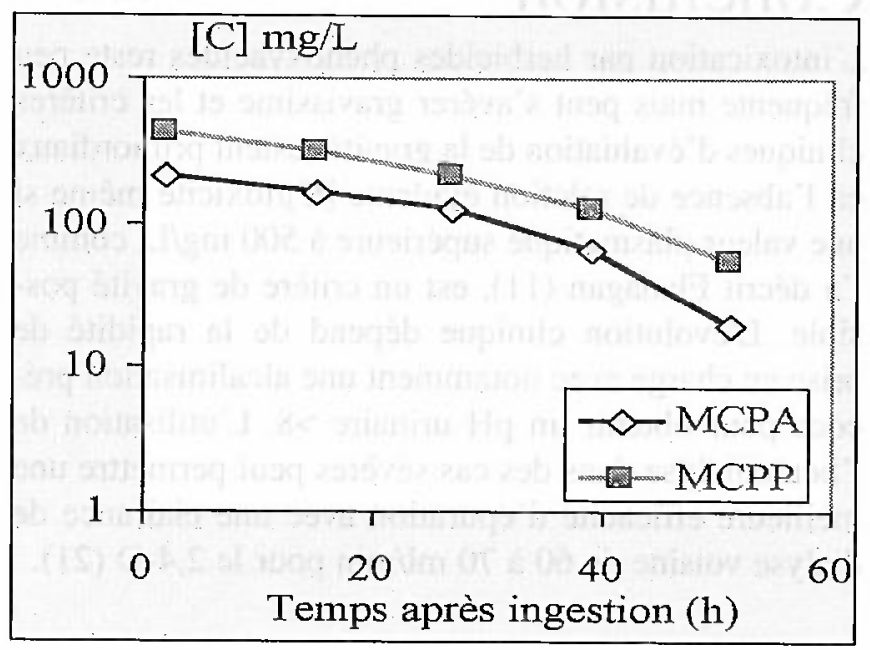

Figure 4 : Cinétiques du MCPA et MCPP observées dans un. cas d'intoxication non mortelle (MCPA $=210 \mathrm{mg} / \mathrm{L}$ et $M C P P=470 \mathrm{mg} / \mathrm{L}$ à $H 2$; Poison Severity Score $=1$ ) après alcalinisation importante et précoce $(60 \mathrm{meq}$ puis $500 \mathrm{meg}$ en 48h, pH urine $>8,5$ ). voisine de $18 \mathrm{~h}$ ) et augmente considérablement dès la $10^{\circ}$ heure pour le 2,4-D ou à partir de la $14^{*}$ heure $(\mathrm{pH}>9)$ pour le MCPP (T1/2 voisine de $4 \mathrm{~h}$ ). Les clairances rénales pour le 2,4-D et le MCPP sont égales à 0,23 et $0,64 \mathrm{ml} / \mathrm{min}$ pendant les dix premières heures et augmentent respectivement à 33,6 et $8,2 \mathrm{ml} / \mathrm{min}$ entre la $14^{\circ}$ et la $18^{\mathrm{e}}$ heure quand le $\mathrm{pH}$ urinaire est égal à 9,6 .

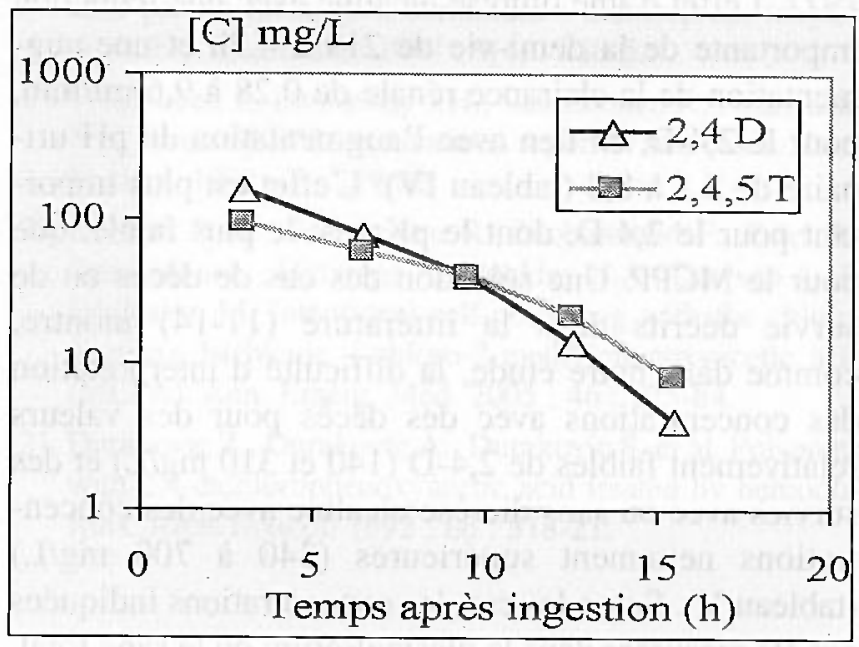

Figure 5 : Cinétiques du 2,4-D et 2,4,5-T observées dans un cas d'intoxication bénigne $(2,4-D=155 \mathrm{mg} / \mathrm{L}$ et 2,4,5-T = $92 \mathrm{mg} / \mathrm{L}$ à H3) après alcalinisation précoce ( $\mathrm{pH}$ urine $>9$ ).

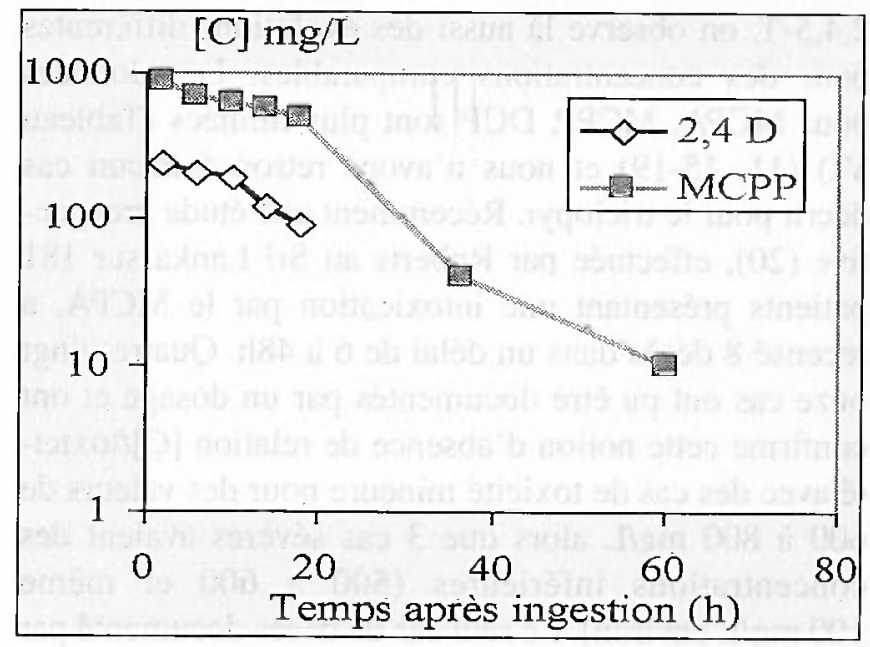

Figure 6 : Cinétiques du 2,4-D et MCPP observées dans un cas d'intoxication bénigne $(2,4-D=240 \mathrm{mg} / L$ et $M C P P=$ $870 \mathrm{mg} / \mathrm{L}$ à H2). $\mathrm{pH}$ urine $<6$ pendant lOh et $>9$ à partir. de 181 . 


\section{Discussion}

Ce travail décrit une étude rétrospective clinico-biologique où n'ont été retenues que les 27 intoxications validées par des dosages plasmatiques. Bradberry (3) a fait en 2004 une revue exhaustive des cas d'intoxications décrits entre 1962 et 2003 avec 23 décès sur 69 cas rapportés. Comme dans notre étude, le 2,4-D est la molécule la plus souvent décrite $(n=56)$, seule ou en association, ce qui rend difficile l'interprétation des toxicités relatives de ces différents herbicides. De plus le rôle des solvants et surfactants est également évoqué. Les 4 cas mortels de notre série avaient tous les critères cliniques pronostiques comportant les troubles de la conscience, une hypotension et une acidose métabolique dès la prise en charge médicale. Tous les survivants avaient un $\mathrm{pH}$ sanguin supérieur à 7,30.

Les herbicides chlorophénoxy- étant des acides faibles de $\mathrm{pKa}$ compris entre 2,64 et 4,84 , Park (5) a décrit, dès 1977, l'effet d'une diurèse alcaline avec une réduction importante de la demi-vie de 219 à 4,7h et une augmentation de la clairance rénale de 0,28 à $9,6 \mathrm{ml} / \mathrm{min}$, pour le 2,4-D, en lien avec l'augmentation du pH urinaire de 5,1 à 8,8 (tableau IV). L'effet est plus important pour le 2,4-D, dont le pKa est le plus faible, que pour le MCPP. Une sélection des cas de décès ou de survie décrits dans la littérature (11-14) montre, comme dans notre étude, la difficulté d'interprétation des concentrations avec des décès pour des valeurs relativement faibles de $2,4-\mathrm{D}(140$ et $310 \mathrm{mg} / \mathrm{L})$ et des survies avec ou sans diurèse alcaline avec des concentrations nettement supérieures (340 à 700 mg/L) (tableau V). Selon les cas, les concentrations indiquées ont été mesurées dans le plasma/sérum ou le sang total. Le ratio plasma/sang total est estimé à 1,7 à 2 (11-12), traduisant une faible diffusion globulaire. En cas d'association de 2,4-D et de MCPP, de dichlorprop ou de 2,4,5-T, on observe là aussi des évolutions différentes pour des concentrations comparables. Les données pour MCPA, MCPP, DCP sont plus limitées (Tableau VI) $(11,15-19)$ et nous n'avons retrouvé aucun cas décrit pour le triclopyr. Récemment une étude prospective (20), effectuée par Roberts au Sri Lanka sur 181 patients présentant une intoxication par le MCPA, a recensé 8 décès dans un délai de 6 à 48 h. Quatre-vingt onze cas ont pu être documentés par un dosage et ont confirmé cette notion d'absence de relation [C]/toxicité avec des cas de toxicité mineure pour des valeurs de 600 à $800 \mathrm{mg} / \mathrm{L}$ alors que 3 cas sévères avaient des concentrations inférieures (500 à 600 et même $100 \mathrm{mg} / \mathrm{L}$ environ). Le seul cas de décès documenté par un dosage avait une valeur voisine de $100 \mathrm{mg} / \mathrm{L}$.
Tableau IV : Relation entre clairance rénale, demi-vie et $\mathrm{pH}$ urinaire d'après (5).

\begin{tabular}{|c|c|c|c|c|}
\hline Urine pH & $\begin{array}{c}2,4-D \\
\text { Cr (mi/min) }\end{array}$ & $\begin{array}{c}2,4-D \\
\text { TU/2 (h) }\end{array}$ & $\begin{array}{c}\text { MCPP } \\
\text { Clr (mil/min) }\end{array}$ & $\begin{array}{c}\text { MCPP } \\
\text { TH2 (h) }\end{array}$ \\
\hline $5,1-6,5$ & 0,28 & 219 & 0,38 & 39 \\
\hline $6,55-7,5$ & 1,14 & 42 & 0,65 & 22 \\
\hline $7,55-8,8$ & 9,60 & 4,7 & 2,08 & 14 \\
\hline
\end{tabular}

Tableau V: Concentrations plasmatiques (sauf st = sang total) en herbicides décrites lors d'intoxications par le 2,4$D$ seul ou en association (11-14).

\begin{tabular}{|c|c|c|c|c|c|c|}
\hline Herbicide(s) & C (mg/t) & & & délai & Herbicide associé & Réf. \\
\hline \multirow[t]{6}{*}{ 2,4-D } & 140 & $\mathrm{D}$ & & $<8 \mathrm{~h}$ & Ioxynil & (II) \\
\hline & 310 & $\bar{D}$ & & $<8 \mathrm{~h}$ & Ioxynil & (I1) \\
\hline & 360 & $\mathrm{~S}$ & $\mathrm{DA}$ & & Ioxynil, Dicamba & (11) \\
\hline & 670 & $S$ & $\mathrm{DA}$ & & Dicamba & (11) \\
\hline & 700 & $S$ & & & Dicamba & (11) \\
\hline & $750\left(389^{\prime \prime}\right)$ & $\bar{D}$ & & $2 j$ & & (12) \\
\hline \multirow[t]{3}{*}{ 2,4-D/MGPP } & $200 / 400^{x / 2}$ & $\mathrm{D}$ & & $18 \mathrm{~h}$ & & (11) \\
\hline & $400 / 751$ & 5 & $\mathrm{DA}$ & & & (13) \\
\hline & $520 / 530^{\mathrm{s}}$ & $\bar{D}$ & & $5 \mathrm{~h}$ & Dicamba & (14) \\
\hline \multirow[t]{2}{*}{ 2,4-D/DCP } & $200 / 400$ & $\bar{D}$ & $\overline{D A}$ & $28 \mathbf{j}$ & & (II) \\
\hline & $240 / 600$ & $S$ & & & & (11) \\
\hline $2,4-D / 2,4,5-T$ & $600 / 400$ & $\mathrm{~S}$ & $\overline{D A}$ & & & (11) \\
\hline
\end{tabular}

$\mathrm{D}=$ décès ; $\mathrm{S}=$ survie $; \mathrm{DA}=$ diurèse alcaline ; délai entre ingestion et décès.

Tableau VI : Concentrations plasmatiques (pl) ou dans le sang total (st) décrites lors d'intoxications par herbicides autres que le 2,4-D (11,15-19).

\begin{tabular}{|c|c|c|c|c|c|c|}
\hline Herbicide(s) & C (mg/L) & & & délai & Herbicide associe & Ref: \\
\hline \multirow[t]{4}{*}{ MCPA } & $320^{\infty}$ & D & & $5 j$ & Dicamba & (11) \\
\hline & $230^{ \pm}$ & $\overline{\mathrm{D}}$ & & $20 \mathrm{~h}$ & 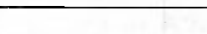 & (15) \\
\hline & $180^{s a}$ & $\mathrm{D}$ & & $20 \mathrm{~h}$ & & (16) \\
\hline & $546^{\mathrm{F}}$ & $S$ & DA & & & (17) \\
\hline$\overline{\mathrm{DCP}}$ & $410^{s t}$ & $\overline{\mathrm{D}}$ & & $\mathrm{I}, 5 \mathrm{~h}$ & Ioxynil, Dicamba & (18) \\
\hline \multirow[t]{2}{*}{ MCPP } & $400^{\mathrm{m}}$ & $S$ & & & Ioxynil, EG & (11) \\
\hline & $298^{\text {ps }}$ & $S$ & & & & (19) \\
\hline
\end{tabular}

$\mathrm{D}=$ décès $; \mathrm{S}=$ survie $; \mathrm{DA}=$ diurèse alcaline ; délai entre ingestion et décès $; \mathrm{EG}=$ Ethylène Glycol.

\section{Conclusion}

L'intoxication par herbicides phénoxyacides reste peu fréquente mais peut s'avérer gravissime et les critères cliniques d'évaluation de la gravité restent primordiaux en l'absence de relation évidente [C]/toxicité même si une valeur plasmatique supérieure à $500 \mathrm{mg} / \mathrm{L}$, comme l'a décrit Flanagan (11), est un critère de gravité possible. L'évolution clinique dépend de la rapidité de prise en charge avec notamment une alcalinisation précoce pour obtenir un $\mathrm{pH}$ urinaire $>8$. L'utilisation de l'hémodialyse dans des cas sévères peut permettre une meilleure efficacité d'épuration avec une clairance de dialyse voisine de 60 à $70 \mathrm{ml} / \mathrm{min}$ pour le 2,4-D (21). 


\section{Références}

1. Poisindex, Micromedex Healthcare series, Chicago, 2006 Vol 127.

2. Bradberry S.M., Watt B.E., Proudfoot H., Vale J.A. Mechanisms of toxicity, clinical features and management of acute chlorophenoxy herbicide poisoning : a review. Clin. Toxicol. 2000; 38:111-22.

3. Bradberry S.M., Proudfoot A.T., Vale J.A. Poisoning due to chlorophenoxy herbicides. Toxicol. Rev. 2004, $23: 65$ 73.

4. Arnold E.K., Beasley V.R. The pharmacokinetics of chlorinated phenoxy acid herbicides : a literature review. Vet. Hum. Toxicol. $1989 ; 31: 121-5$.

5. Park J., Darrien I., Prescott L.F. Pharmacokinetic studies in severe intoxication with 2,4-D and mecoprop. Proc. Eur. Soc. Toxicol. $1977 ; 18: 154-5$.

6. Personn H.E., Sjöberg G.K., Haines J.A., Pronczuk de Garbino J. Poisoning severity score. Grading of acute poisoning. Clin. Toxicol. $1998 ; 36: 205-13$.

7. Turcant A., Dehon B., Ganière-Monteil C., Dulaurent S., Moulsma M., Charlier C. Analyse des herbicides phénoxyacides par chromatographie en phase liquide : $1^{\text {er }}$ essai interlaboratoires. Ann. Tox. Anal. 2006 ; 18(2) : 135-42.

8. Blanchet J.P., Turcant A., Harry P., Durand R., Allain P. Intoxication mortelle par le LONPAR. Ann. Tox. Anal. $2000 ; 12(2): 169$.

9. Ganière-Monteil C., Kergueris M.F., Rodineau P., Harry P., Dailly E., Pineau A., Azoulay C., Bourin M. Intoxication mortelle au Dichlorprop, MCPA, Sulfosate. Ann. Tox. Anal. 2001 ; 13(4) : 290-1.

10. Ganière-Monteil C., Kergueris M.F., Lamiable D., Pineau A., Azoulay C., Bourin M. Intoxication mortelle par la Desormone. Ann. Tox. Anal. $2000 ; 12(2)$ : 163.

11. Flanagan R.J, Meredith T.J., Muprah M., Onyon L.J., Liddle A. Alkaline diuresis for acute poisoning with chlorophenoxy herbicides and ioxynil. Lancet $1990 ; 335$ : 454-9.
12. Keller T., Skopp G., Wu M., Aderjan R. Fatal overdose of 2,4-dichlorophenoxyacetic acid (2,4-D). Forensic Sci. Int. $1994 ; 65: 13-8$.

13. Prescott L.F., Park J., Darrien I. Treatment of severe 2,4-D and mecoprop intoxication with alkaline diuresis. Br. J. Clin. Pharm. $1979 ; 7: 111-6$.

14. Fraser A.D., Isner A.F., Perry R.A. Toxicological studies in a fatal overdose of $2,4-\mathrm{D}$, mecoprop and dicamba. J. Forensic Sci. $1984 ; 29:$ 1237-44.

15. Popham R.D., Davies D.M. A case of MCPA poisoning. Br. Med. J. 1964 ; 1: 677-8.

16. Johnson H.R., Koumides O. A further case of MCPA poisoning. Br. Med. J. $1965 ; 2$ : 629-30.

17. Schmoldt A., Iwersen S., Schlüter W. Massive ingestion of the herbicide 2-methyl-4-chlorophenoxyacetic acid (MCPA). Clin. Toxicol. 1997 ; 35 : 405-8.

18. Richard N., Salvadore O., Ghysel M.H. Intoxication mortelle par un mélange d'herbicides : dichlorprop, ioxyail, dicamba. Toxicorama $1997 ; 9(4): 280-5$.

19. Meulenbelt J., Swaveling J.H., Van Zoonen P., Notermans N.C. Acute MCPP intoxication : report of two cases. Hum. Toxicol. $1988 ; 7: 289-92$.

20. Roberts D.M., Seneviratne R., Mohammed F., Patel R., Senerathna L., Hittarage A., Buckley N.A., Dawson A.H., Eddleston M. Intentional self-poisoning with the chlorophenoxy herbicide 4-chloro-2-methylphenoxyacetic acid (MCPA). Ann. Emerg. Med. 2005 ; 46 : 275-84.

21. Durakovic Z., Durakovic A., Durakovic S. et al. Poisoning with 2,4-dichlorophenoxyacetic acid treated by hemodialysis. Arch.Toxicol. $1992 ; 66: 518-21$. 